\title{
Protective Effect of Antrodia Camphorate on the Tissues of Brain, Kidney, Lung, and Liver Is Correlated with Declining Lipid Peroxidation
}

\author{
Mei-Min Shiu ${ }^{1}$, Bo-Yu Huang ${ }^{2}$, Chien-Chi Liu ${ }^{3}$, Chin-Sheng Liao², Ming-Cheng Lin ${ }^{2, *}$ \\ ${ }^{1}$ Division of Hematology/Medical Oncology, Department of Medicine, Taichung Veterans General Hospital, Taichung, Taiwan \\ ${ }^{2}$ Department of Medical Laboratory Science and Biotechnology, Central Taiwan University of Science and Technology, Taichung, Taiwan \\ ${ }^{3}$ Department of Nursing, Hung Kuang University, Taichung, Taiwan
}

\section{Email address:}

mclin@ctust.edu.tw (Ming-Cheng Lin)

${ }^{*}$ Corresponding author

\section{To cite this article:}

Mei-Min Shiu, Bo-Yu Huang, Chien-Chi Liu, Chin-Sheng Liao, Ming-Cheng Lin. Protective Effect of Antrodia Camphorate on the Tissues of Brain, Kidney, Lung, and Liver Is Correlated with Declining Lipid Peroxidation. American Journal of Laboratory Medicine.

Vol. 3, No. 1, 2018, pp. 6-10. doi: 10.11648/j.ajlm.20180301.12

Received: September 23, 2017; Accepted: October 16, 2017; Published: December 14, 2017

\begin{abstract}
Previous investigation has proposed that Antrodia Camphorata possesses beneficial effect mainly on liver protection. To date, the antioxidative effect of Antrodia Camphorata on the tissues of brain, kidney, lung, and liver, however, has not yet clarified. In this current experiment, eighty male Sprague-Dawley rats were randomly divided into control and Antrodia Camphorate-treated subject. Both subjects were intraperitoneally injected with normal saline and Antrodia Camphorata for consecutive 14 days, respectively. On day 15, rats were sacrificed and tissues of brain, kidney, lung, and liver were immediately harvested and homogenate. The malondialdehyde level, an end-product of lipid peroxidation, was measured in different tissues. Experimental result showed that the malondialdehyde levels were significantly $(\mathrm{P}<0.05)$ reduced in all tissues after receiving Antrodia Camphorate as compared with the control subject. Specifically, an obvious decline (12.93\%) of the malondialdehyde level was found in the brain as compared to the tissues of kidney, lung, and liver. Accordingly, our present result indicates that Antrodia Camphorate can significantly decline oxidative damage as presented by a reduced malondialdehyde levels in the tissues of brain, kidney, lung, and liver. In addition, it seems likely that the best tissue protective efficacy offered by Antrodia Camphorate in declining oxidative damage is found in the brain tissue.
\end{abstract}

Keywords: Antrodia Camphorata, Lipid Peroxidation, Antioxidative Effect, Malondialdehyde

\section{Introduction}

It has been previously proposed that Antrodia Camphorata (AC), one of the traditional Chinese Medicines, possesses beneficial effects in treating a variety of human diseases such as diarrhea, hypertension, abdominal pain, chemical intoxication, and is mainly used for the treatment of liver disease in most of the Asia countries such as Taiwan [1-3]. Investigation has revealed that Antrodia Camphorata can exert its immune-modulatory ability and hepatoprotection due to its antioxidant property [4-5]. Previous study has suggested that Antrodia Camphorata can scavenge deleterious free radical molecules mediated by chemical- induced hepatotoxicity [6]. Furthermore, in vivo study has indicated that Antrodia Camphorata not only can elevate hepatic glutathione-dependent enzymes upon protecting chemical-induced lesion on rat liver but also may decline oxidative injury conducted by toxic molecules of hydrogen peroxide [7]. On the other hand, previous experiment has indicated that cerebral ischemia-induced brain infarction and neurobehavioral disorder can be improved via administration of the traditional Chinese Medicine of Antrodia Camphorata [8-12]. As mentioned above, it seems likely to emphasize its beneficial effect here that Antrodia Camphorate may exactly 
exert its excellent antioxidant ability to alleviate further oxidative lesion.

Deleterious lipid peroxidation is a complex reactive oxygen species (ROS) inducing reaction [13]. Due to the utilization of aerobic metabolism in most of the living organisms, large amount of the generated ROS may attack the composition of polyunsaturated fatty acid (PUFA) and result in further adversely oxidative effect in cells [14]. Under this circumstance, elevated oxidative stress and cellular injury occur [15]. Malondialdehyde (MDA) is a wellknown stable end-product of lipid peroxidation. Moreover, magnitude of lipid peroxidation is also well-recognized in paralleled with the concentration of MDA, the end-product of lipid peroxidation [16-18]. For this reason, elevated MDA level not only represents increased lipid peroxidation level but also is parallel with the severity of human diseases.

Large amount of the research papers have recently focused on investigating its beneficial effect of Antrodia Camphorata on the liver disease. However, whether this positive effect can be observed except for the liver tissue, however, has not yet been clarified and needs to be further elucidated.

\section{Materials and Methods}

\subsection{Experimental Animal Treatment}

Experimentally, eighty male Sprague-Dawley rats, weighing from 220-270 g were encompassed in this present work. All experimental rats were purchased from National Laboratory Animal Breeding and Research Center (Taiwan). All rats were kept in stainless-steel mesh cages, housed under controlled conditions $\left(22 \pm 2^{\circ} \mathrm{C}, 50 \pm 20 \%\right.$ relative humidity, 12-h light-dark cycle) with diet and water. All animal handling and experimental protocols were approved by the Institutional Animal Care and Use Committee (IACUC) of Central Taiwan University of Science and Technology. All experimental rats were randomly divided into control and Antrodia Camphorate-administrated group of 10 rats each. Control and Antrodia Camphorate-treated rats were intraperitoneally injected with $1 \mathrm{ml} / \mathrm{kg}$ of isotonic normal saline and Antrodia Camphorata $(250 \mathrm{mg} / \mathrm{Kg})$ once in a day for consecutive 14 days, respectively.

\subsection{Measurement of the Malondialdehyde Level in the Tissues of Brain, Lung, Liver, and Kidney}

On day 15, all rats were sacrificed and the tissues including brain, lung, liver, and kidney were isolated for further biochemical analysis. In brief, the isolated tissues $(0.5$ $\mathrm{g}$, wet weight) was immediately homogenized in $5 \mathrm{ml}$ of cold $\mathrm{KCl}(154 \mathrm{mM})$ solution using motor driven tissue homogenizers with Teflon pestles and then centrifuged at $4^{\circ} \mathrm{C}$ for $15 \mathrm{~min}$ at $10000 \mathrm{~g}$. The supernatants were harvested for the measurement of the level of malondialdehyde. For malondialdehyde analysis, the reagent of 1, 1, 3, 3tetraethoxypropane (TEP) was used as a standard solution in the reaction with thiobarbituric acid reactive substance (TBARS). Reagent of thiobarbituric acid (TBA) was purchased from E. Merck (Germany) and used for malondialdehyde analysis. Chemical reagent of 1, 1, 3, 3tetraethoxypropane (TEP) was applied to act as a standard solution in the reaction with thiobarbituric acid reactive substance (TBARS). Generally, the analytical principle of this detective method based on the determination of the pink color that is produced by the interaction of TBA with the component of malondialdehyde. The malondialdehyde level was measured using spectrophotometer (U-1900, Hitachi, Japan) and the wavelength of $532 \mathrm{~nm}$ was selected in this present experiment.

\subsection{Statistical Analysis}

All experimental data were expressed as mean \pm SD. They were statistically analyzed by using Mann-Whitney U Test. Statistical differences were considered significant at a $p$ value less than 0.05 in this present study.

\section{Results}

\subsection{Malondialdehyde Level in the Homogenates of the Brain Tissues}

Our present result showed that the mean MDA value in the control and the Antrodia Camphorata-treated subject was $12.30 \pm 0.34$ and $10.71 \pm 0.36$ ( $\mu$ mole/g protein) respectively. Compared with the control group, a significant reduction $(\mathrm{P}<0.05)$ of the MDA level was observed in Antrodia Camphorata administrated rats as shown in Figure 1.

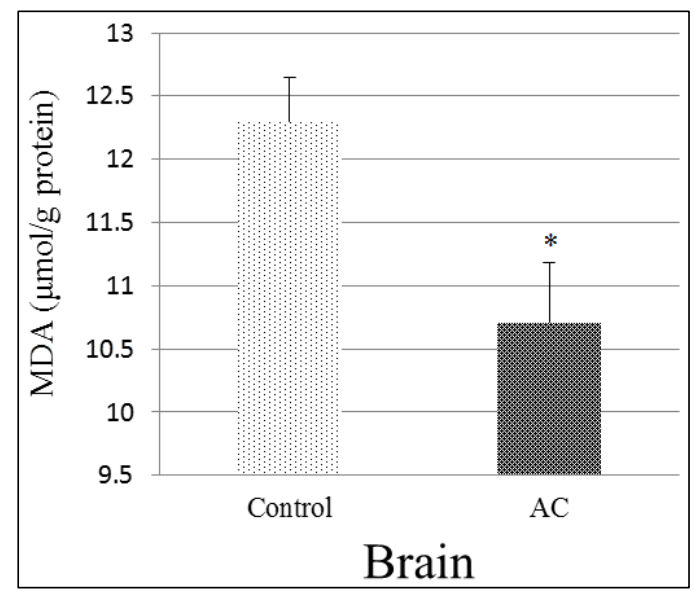

Figure 1. Profiles of the concentration of malondialdehyde (MDA) in brain tissues. Data are expressed as mean $\pm S D(n=10)$. The MDA value in control and Antrodia Camphorata-treated subject was $12.30 \pm 0.34$ and $10.71 \pm$ 0.36 ( $\mu$ mole/g protein) respectively. $* p<0.05$ versus control group as determined by Mann-Whitney U test.

\subsection{Malondialdehyde Level in the Homogenates of the Lung Tissues}

It has also been considered that the intensity of lipid peroxidation is positively correlated with the MDA level. Given the fact, higher MDA level represents higher lipid peroxidation and oxidative stress. Our present observation indicated that the MDA level in the control and the Antrodia 
Camphorata-treated subject was $8.80 \pm 0.46$ and $8.40 \pm 0.13$ ( $\mu$ mole/g protein) respectively. Compared with the control group, a significant reduction $(\mathrm{P}<0.05)$ of which level was found in Antrodia Camphorata administrated rats as shown in Figure 2.

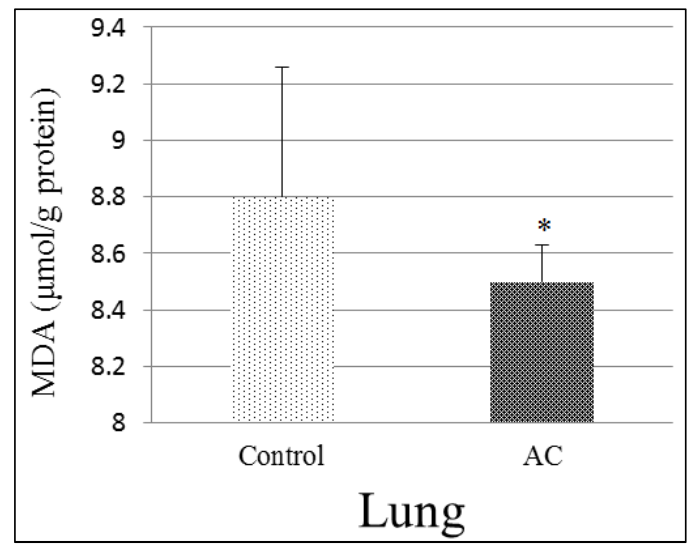

Figure 2. Profiles of the concentration of malondialdehyde (MDA) in lung tissues. Data are expressed as mean $\pm S D(n=10)$. The MDA value in control and Antrodia Camphorata-treated subject was $8.80 \pm 0.46$ and $8.40 \pm 0.13$ ( $\mu$ mole/g protein) respectively. ${ }^{*} p<0.05$ versus control group as determined by Mann-Whitney U test.

\subsection{Malondialdehyde Level in the Homogenates of the Liver Tissues}

It has also been recognized that intensity of lipid peroxidation is positively correlated with the MDA level. That is to say, higher MDA level represents higher lipid peroxidation and oxidative injury. Our present observation indicated that the MDA level in the control and the Antrodia Camphorata-treated subject was $13.76 \pm 0.42$ and $13.33 \pm$ 0.14 ( $\mu$ mole/g protein) respectively. Compared with the control group, a significant reduction $(\mathrm{P}<0.05)$ of the MDA level was seen in Antrodia Camphorata administrated rats as listed in Figure 3.

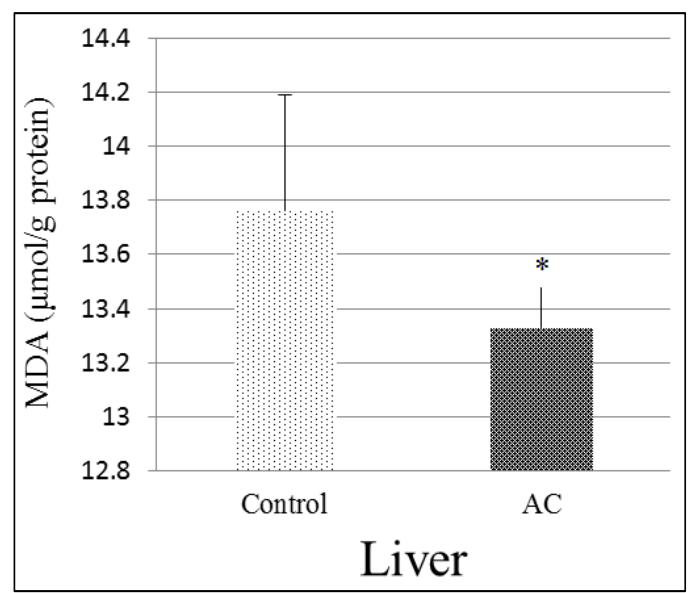

Figure 3. Profiles of the concentration of malondialdehyde (MDA) in liver tissues. Data are expressed as mean $\pm S D(n=10)$. The MDA value in control and Antrodia Camphorata-treated subject was $13.76 \pm 0.42$ and $13.33 \pm$ 0.14 (umole/g protein) respectively. $*_{p}<0.05$ versus control group as determined by Mann-Whitney U test.

\subsection{Malondialdehyde Level in the Homogenates of the Kidney Tissues}

It has also been considered that the intensity of lipid peroxidation is positively associated with the malondialdehyde level. Given the fact, higher MDA level represents higher lipid peroxidation and oxidative stress. Our present observation indicated that MDA level in the control and the Antrodia Camphorata-treated subject was $12.98 \pm$ 0.39 and $12.36 \pm 0.13$ ( $\mu$ mole/g protein) respectively. Compared with the control group, a significant reduction $(\mathrm{P}<0.05)$ of the MDA concentration was observed in Antrodia Camphorata-treated rats as listed in Figure 4.

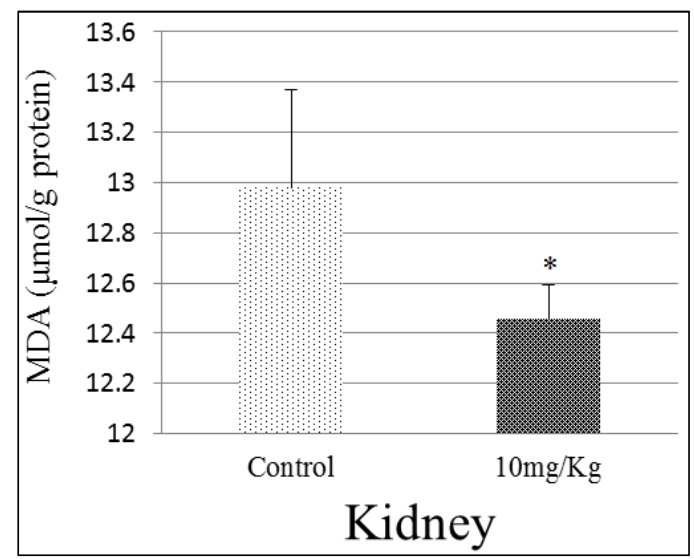

Figure 4. Profiles of the concentration of malondialdehyde (MDA) in kidney tissues. Data are expressed as mean $\pm S D(n=10)$. The MDA value in control and Antrodia Camphorata-treated subject was $12.98 \pm 0.39$ and $12.36 \pm$ 0.13 (umole/g protein) respectively. $* p<0.05$ versus control group as determined by Mann-Whitney U test.

\section{Discussion}

It has been realized that aerobic organisms, which derive their energy from the reduction of oxygen, are susceptible to reactive oxygen species (ROS) such as superoxide radicals, hydrogen peroxide, and hydroxyl radicals that inevitably form during the metabolism of oxygen. Also, once large amount of the ROS are generated, these toxic molecules may spontaneously react with the composition of poly-unsaturated fatty acid (PUFA) in cells and given the fact, further oxidative lesion named lipid peroxidation occurs. In addition, further investigation has proposed the fact that a number of diseases have been documented in associated with increased lipid peroxidation due to elevated ROS level [1-2]. The Chinese Medicine of Antrodia Camphorata has been mainly used for the treatment of liver disease, immune-dysfunction, food poisoning, drug intoxication, diarrhea, abdominal pain, hypertension, skin irritation and cancer [1-3]. This medicinal mushroom is starting to attract interest due to it possesses a variety of bioactive compounds such as polysaccharides, triterpenoids, maleic/succinic acid derivatives and benzenoids derivatives [2-4]. Increasing evidence has proposed that Antrodia Camphorata possesses multiple ranges of biological efficacies including, immunemodulation, anti-oxidation, anti-inflammation, hepatoprotection, anti-cancer, and anti-hypertension [3-6]. In vivo study has 
revealed that Antrodia Camphorata exerts anti-oxidation effects against $\mathrm{H}_{2} \mathrm{O}_{2}$-induced cytotoxicity in cells in carbon tetrachloride-induced hepatotoxicity [7]. Additionally, Antrodia Camphorata plays an important role in preventing oxidative lesion by up-regulating the hepatic glutathione-dependent enzymes in living systems to preserve the normal reduced and oxidized glutathione (GSH/GSSH) ratio has been reported [5]. Investigation has pointed out that mycelia of Antrodia Camphorata could exhibit anti-hepatitis B virus effect [8]. Furthermore, it has been reported previously that Antrodia Camphorata could inhibit N-formyl-methionylleucylphenylalanine (fMLP) or phorbol 12-myristate 13- acetate(PMA-) induced ROS production in peripheral human neutrophils [6]. Our present study clearly showed that Antrodia Camphorata can significantly reduce lipid peroxidation level in the brain cortex of rats as compared with the control group (Figure 1). In fact, lipid peroxidation processes are also known a complex ROS inducing reaction caused by the degradation of polyunsaturated fatty acid (PUFA) in cells. In this regard, higher MDA level represents elevated oxidative stress and injury has been well-recognized. Our present observation indicated that Antrodia Camphorata can significantly inhibit free radicalmediated lipid peroxidation, and our present finding was in according with the previous investigation $[9-13,18]$.

\section{Conclusion}

Our results clearly show that traditional Chinese Medicine of Antrodia Camphorate can significantly reduce the malondialdehyde levels in the tissues of the brain, liver, lung, and kidney. Meanwhile, an obvious reduction of the malondialdehyde levels was found in the brain tissue as compared to other tissues. In conclusion, our current observations manifest that Antrodia Camphorata possesses the protective efficacy in attenuating reactive oxygen species mediated oxidative tissue injury. Furthermore, our experimental finding demonstrates that the best tissue protective efficacy produced by Antrodia Camphorate is found in the brain tissue and possibly, Antrodia Camphorata may be can considered an useful medicinal potential in preventing and treating the brain tissues from further oxidative lesion such as cerebral ischemic insult.

\section{Acknowledgements}

The authors would gratefully acknowledge Central Taiwan University of Science and Technology (CTU105-PC-003) for financial support.

\section{References}

[1] Shen, Y. C., Chou, C. J., Wang, Y. H., Chen, C. F., Chou, Y. C., \& Lu, M. K. (2004). Anti-inflammatory activity of the extracts from mycelia of Antrodia camphorata cultured with water-soluble fractions from five different Cinnamomum species. FEMS Microbiol Lett, 231 (1), 137-143.
[2] Lee, J. J., Hsu, W. H., Yen, T. L., Chang, N. C., Luo, Y. J., Hsiao, G., \& Sheu, J. R. (2011). Traditional Chinese medicine, $\mathrm{Xue}-\mathrm{Fu}-\mathrm{Zhu}-\mathrm{Yu}$ decoction, potentiates tissue plasminogen activator against thromboembolic stroke in rats. J Ethnopharmacol, 134 (3), 824-830.

[3] Cuzzocrea, S., Riley, D. P., Caputi, A. P., \& Salvemini, D. (2001). Antioxidant therapy: a new pharmacological approach in shock, inflammation, and ischemia/reperfusion injury. Pharmacological reviews, 53 (1), 135-159.

[4] Hsiao, G., Shen, M.-Y., Lin, K.-H., Lan, M.-H., Wu, L.-Y., Chou, D.-S., Sheu, J.-R. (2003). Antioxidative and hepatoprotective effects of Antrodia camphorata extract. Journal of agricultural and food chemistry, 51 (11), 33023308 .

[5] Cheng, P.-C., Hsu, C.-Y., Chen, C.-C., \& Lee, K.-M. (2008). In vivo immunomodulatory effects of Antrodia camphorata polysaccharides in a T1/T2 doubly transgenic mouse model for inhibiting infection of Schistosoma mansoni. Toxicology and Applied Pharmacology, 227 (2), 291-298.

[6] Hseu, Y.-C., Yang, H.-L., Lai, Y.-C., Lin, J.-G., Chen, G.-W., \& Chang, Y.-H. (2004). Induction of apoptosis by Antrodia camphorata in human premyelocytic leukemia HL-60 cells. Nutrition and Cancer, 48 (2), 189-197.

[7] Song, T.-Y., \& Yen, G.-C. (2003). Protective effects of fermented filtrate from Antrodia camphorata in submerged culture against $\mathrm{CCl}_{4}$-induced hepatic toxicity in rats. Journal of agricultural and food chemistry, 51 (6), 1571-1577.

[8] Hsu, Y.-L., Kuo, Y.-C., Kuo, P.-L., Ng, L.-T., Kuo, Y.-H., \& Lin, C.-C. (2005). Apoptotic effects of extract from Antrodia camphorata fruiting bodies in human hepatocellular carcinoma cell lines. Cancer letters, 221 (1), 77-89.

[9] Malik, Z. A., Singh, M., \& Sharma, P. (2011). Neuroprotective effect of Momordica charantia in global cerebral ischemia and reperfusion induced neuronal damage in diabetic mice. Journal of Ethnopharmacology, 133 (2), 729-734.

[10] Lee, Y.-M., Chang, C.-Y., Yen, T.-L., Geraldine, P., Lan, C.C., Sheu, J.-R., \& Lee, J.-J. (2014). Extract of Antrodia camphorata exerts neuroprotection against embolic stroke in rats without causing the risk of hemorrhagic incidence. The Scientific World Journal, 2014.

[11] Hsiao, G., Lin, K. H., Chang, Y., Chen, T. L., Tzu, N. H., Chou, D. S., \& Sheu, J. R. (2005). Protective mechanisms of inosine in platelet activation and cerebral ischemic damage. Arteriosclerosis, thrombosis, and vascular biology, 25 (9), 1998-2004.

[12] Huang, F.-P., Xi, G., Keep, R. F., Hua, Y., Nemoianu, A., \& Hoff, J. T. (2002). Brain edema after experimental intracerebral hemorrhage: role of hemoglobin degradation products. Journal of neurosurgery, 96 (2), 287-293.

[13] Nazıroğlu, M., Kutluhan, S., Uğuz, A. C., Çelik, Ö., Bal, R., \& Butterworth, P. J. (2009). Topiramate and vitamin E modulate the electroencephalographic records, brain microsomal and blood antioxidant redox system in pentylentetrazol-induced seizure of rats. Journal of Membrane Biology, 229 (3), 131-140.

[14] Limón-Pacheco, J., \& Gonsebatt, M. E. (2009). The role of antioxidants and antioxidant-related enzymes in protective responses to environmentally induced oxidative stress. Mutation Research/Genetic Toxicology and Environmental Mutagenesis, 674 (1), 137-147. 
[15] Sun, A. Y., Wang, Q., Simonyi, A., \& Sun, G. Y. (2008). Botanical phenolics and brain health. Neuromolecular medicine, 10 (4), 259-274.

[16] Özcelik, D., Uzun, H., \& Nazıroglu, M. (2012). Nacetylcysteine attenuates copper overload-induced oxidative injury in brain of rat. Biological trace element research, 147 (1-3), 292-298.
[17] Meneghini, R. (1997). Iron homeostasis, oxidative stress, and DNA damage. Free Radical Biology and Medicine, 23 (5), 783-792.

[18] Fang, K.-M., Cheng, F.-C., Huang, Y.-L., Chung, S.-Y., Jian, Z.-Y., \& Lin, M.-C. (2013). Trace element, antioxidant activity, and lipid peroxidation levels in brain cortex of gerbils after cerebral ischemic injury. Biological trace element research, 152 (1), 66-74. 